OPEN ACCESS

Edited by:

José M. Álvarez-Castro,

University of Santiago

de Compostela, Spain

Reviewed by:

Filippo Biscarini,

National Research Council (CNR), Italy

Jin Ok Yang,

Korean Bioinformation Center, Korea

Research Institute of Bioscience and Biotechnology, South Korea

*Correspondence:

Mohammad Taheri

Mohammad_823@yahoo.com

Soudeh Ghafouri-Fard

s.ghafourifard@sbmu.ac.ir

Specialty section:

This article was submitted to Applied Genetic Epidemiology,

a section of the journal

Frontiers in Genetics

Received: 01 June 2021

Accepted: 12 August 2021

Published: 16 September 2021

Citation:

Miladipour A, Gholipour M Honarmand Tamizkar K, Abak A, Kholghi Oskooei V, Taheri M and Ghafouri-Fard S (2021) Investigation

of FADS Gene Cluster Single

Nucleotide Polymorphisms

in End-Stage Renal Disease

Compared With Normal Controls.

Front. Genet. 12:716151.

doi: 10.3389/fgene.2021.716151

\section{Investigation of FADS Gene Cluster Single Nucleotide Polymorphisms in End-Stage Renal Disease Compared With Normal Controls}

\begin{abstract}
Amirhossein Miladipour1, Mahdi Gholipour'2, Kasra Honarmand Tamizkar², Atefe Abak ${ }^{3}$, Vahid Kholghi Oskooei ${ }^{4,5}$, Mohammad Taheri6* and Soudeh Ghafouri-Fard ${ }^{7 *}$

${ }^{1}$ Urology and Nephrology Research Center, Shahid Beheshti University of Medical Sciences, Tehran, Iran, ${ }^{2}$ Men's Health and Reproductive Health Research Center, Shahid Beheshti University of Medical Sciences, Tehran, Iran, ${ }^{3}$ Phytochemistry Research Center, Shahid Beheshti University of Medical Sciences, Tehran, Iran, ${ }^{4}$ Department of Laboratory Sciences, School of Paramedical Sciences, Torbat Heydariyeh University of Medical Sciences, Torbat Heydariyeh, Iran, ${ }^{5}$ Neuroscience Research Center, Torbat Heydariyeh University of Medical Sciences, Torbat Heydariyeh, Iran, ${ }^{6}$ Skull Base Research Center, Loghman Hakim Hospital, Shahid Beheshti University of Medical Sciences, Tehran, Iran, ${ }^{7}$ Department of Medical Genetics, School of Medicine, Shahid Beheshti University of Medical Sciences, Tehran, Iran
\end{abstract}

End-stage renal disease (ESRD) is a public health problem with a high burden. The condition is associated with abnormalities in lipid metabolism. The fatty acid desaturase (FADS) gene cluster includes three genes that are significantly correlated with a number of pathologic conditions related to abnormal lipid levels. In the current study, we genotyped rs174556, rs99780, and rs7115739 single nucleotide polymorphisms within the FADS cluster in a population of ESRD patients and healthy controls. The rs174556 of the FADS1 gene and rs99780 of the FADS2 gene were not associated with the risk of ESRD in any inheritance model. However, the rs7115739 of FADS3 was associated with the risk of ESRD in all models except for the recessive model. The T allele of this SNP was significantly less prevalent among cases compared with controls [odds ratio (OR) $(95 \% \mathrm{Cl})=0.44(0.25-0.77), P$ value $=0.004]$. GT and $\Pi$ genotypes has been shown to decrease the risk of ESRD in a codominant model [OR $(95 \% \mathrm{Cl})=0.49(0.26-0.92)$ and $\mathrm{OR}(95 \% \mathrm{Cl})=0.18(0.02-1.6)$, respectively; $P$ value $=0.019]$. In the dominant model, GT $+\pi$ status was associated with lower risk of ESRD [OR $(95 \% \mathrm{Cl})=0.45(0.24-0.82)$, $P$ value $=0.0078]$. Assessment of association between this SNP and risk of ESRD in an overdominant model revealed that GT genotype decreases the risk of this condition [OR $(95 \% \mathrm{Cl})=0.5(0.27-0.94), P$ value $=0.029]$. Taken together, the rs7115739 of FADS3 is suggested as a putative modulator of the risk of ESRD in the Iranian population.

Keywords: FADS1, FADS2, FADS3, polymorphisms, hemodialysis

\section{INTRODUCTION}

End-stage renal disease (ESRD) is a public health problem all over the world with an increasing trend in its incidence in both developed and developing countries (Haghighi et al., 2002; Renal Du System (USRDS), 2010; Mousavi et al., 2014). The prevalence of chronic renal disease is estimated to be around 13.4\% throughout the world (Lv and Zhang, 2019). Notably, between 4.902 and 7.083 
million individuals worldwide have ESRD and are in need of kidney transplantation ( $\mathrm{Lv}$ and Zhang, 2019). The total rise in the prevalence of ESRD is mostly due to the rise in the prevalence of a number of associated risk factors including diabetes mellitus and hypertension (Lv and Zhang, 2019). Due to the high burden of this disease and the unavailability of renal replacement therapy in some regions ( $\mathrm{Lv}$ and Zhang, 2019), it is necessary to find the underlying causes and genetic risk factors for the development of ESRD.

Previous studies have shown substantial elevation of the risk of ESRD in patients with a family history of this condition (Satko et al., 2005), implying the presence of genetic risk factors for ESRD. Genome-wide association studies (GWAS) have led to the identification of more than 100 loci for chronic kidney disease and its related conditions (Pattaro et al., 2016; Wuttke and Köttgen, 2016; Gorski et al., 2017). A number of newly identified SNPs have been shown to be associated with transcript levels of SYPL2, SDCCAG8, MANBA, KBTBD2, PTPRO, and SPATA33 genes, thus providing evidence that these genes are potential candidates for the observed associations (Pattaro et al., 2016). Moreover, a number of these loci, including SDCCAG8, LRP2, IGFBP5, SKIL, UNCX, KBTBD2, A1CF, KCNQ1, AP5B1, PTPRO, TP53INP2, and BCAS1 have been shown to be consistently associated with this trait based on random-effect meta-analyses (Pattaro et al., 2016).

The fatty acid desaturase (FADS) gene cluster includes three genes that are significantly correlated with a number of pathologic conditions such as abnormal lipid levels, fatty liver disorder, and diabetes mellitus (Gromovsky et al., 2018). Genetic polymorphisms within the FADS1 and FADS2 genes have been associated with fatty acid metabolic pathways via changing methylation patterns and the expression of genes (He et al., 2018). Moreover, these polymorphisms have been found to affect the plasma levels of fatty acids and desaturase in diabetic patients suffering from coronary artery disease (Li et al., 2016).

End-stage renal disease has been shown to be associated with the premature development of atherosclerosis and mortality from coronary artery diseases due to increased levels of oxidative stress, as well as abnormalities in lipid metabolism (Vaziri, 2009).

Based on the association between ESRD and abnormal lipid metabolism, genes participating in the lipid metabolism might be associated with the development of ESRD. To test this hypothesis, we designed the current study to appraise the association between rs174556, rs99780, and rs7115739 single nucleotide polymorphisms (SNPs) within the FADS cluster on 11q12q13.1 and the risk of ESRD in 164 Iranian patients admitted to Labbafinejad Hospital, Tehran, Iran compared with 171 age- and sex-matched controls.

These SNPs have been selected based on the results of previous studies regarding their role in a number of metabolism-related diseases. The FADS1 rs174556 has been suggested to be associated with acute coronary syndrome in a population of Chinese patients, particularly those with hypertension (Song et al., 2013). Among several SNPs within the FADS cluster, rs174556 has been found to be representative of the disease-associated block in a study (Campoy et al., 2021). rs99780 has been among SNPs associated with alteration of plasma levels of (n-6) and (n-3) essential fatty acids and erythrocyte phospholipids (Xie and Innis, 2008). Finally, rs7115739 has been shown to be associated with total cholesterol levels among Chinese patients (Wu et al., 2017).

\section{MATERIALS AND METHODS}

\section{Patients and Controls}

The current study included a total of 164 cases ( 75 women and 89 men) and 171 controls (81 women and 90 men). Table 1 shows the description of cases and controls.

All cases had severe ESRD. Cases were recruited from the hemodialysis Ward of Labbafinejad Hospital, Tehran, Iran during 2019-2020. Controls were healthy subjects with no personal or familial history of chronic renal disorder. Exclusion criteria were malignancies or autoimmune disorders. Patients with other concurrent disorders were also excluded from the study. The study protocol was approved by the Ethical Committee of Shahid Beheshti University of Medical Sciences (IR. SBMU.UNRC.1398.10). All cases and controls signed the informed consent forms.

\section{Genotyping}

Genomic DNA was isolated from venous blood specimens of all recruited persons using the standard salting-out method (Chacon-Cortes and Griffiths, 2014). The tetra-primer ARMSPCR method (Medrano and de Oliveira, 2014) was used for the genotyping of rs174556, rs99780, and rs7115739 SNPs. For this purpose, $75 \mathrm{ng}$ of genomic DNA, $0.30 \mathrm{pmol}$ of each outer primer, $0.5 \mathrm{pmol}$ of the forward inner primer, $0.80 \mathrm{pmol}$ of the reverse inner primer, and Taq DNA polymerase master mix red (Ampliqon, Denmark). The PCR condition consisted of a preliminary denaturation at $94^{\circ} \mathrm{C}$ for $5 \mathrm{~min}, 35$ cycles of $95^{\circ} \mathrm{C}$ for $45 \mathrm{~s}$, specific annealing temperatures for $40 \mathrm{~s}$, and $72^{\circ} \mathrm{C}$ for $60 \mathrm{~s}$, with the final extension of $72^{\circ} \mathrm{C}$ for 5 min. The rs174556 (NC_000011.9:g.61580635C > T) is an intron variant in the FADS1 gene close to rs981340263 and rs2066943471. The rs99780 (NC_000011.9:g.61596633C > T) is also an intron variant in FADS2 near rs1299703736. Finally, rs7115739 (NC_000011.9:g.61641717T > G) is an intron variant of FADS $3^{1}$. Primer sequences and PCR conditions for genotyping experiments are shown in Table 2.

\section{Statistical Methods}

Genotype and allele frequencies of rs174556, rs99780, and rs7115739 were compared between cases and controls in

\footnotetext{
${ }^{1}$ https://www.ncbi.nlm.nih.gov/snp
}

TABLE 1 | Demographic data of cases and controls.

\begin{tabular}{lcc}
\hline Variables & Cases & Controls \\
\hline Female/Male $[$ no. $(\%)]$ & $75(45 \%) / 89(55 \%)$ & $81(47 \%) / 90(53 \%)$ \\
Age $($ mean $\pm \mathrm{SD}, \mathrm{Y})$ & $56.2 \pm 2.7$ & $52.3 \pm 2.4$ \\
Age range $(\mathrm{Y})$ & $17-85$ & $22-79$
\end{tabular}


TABLE 2 | Primer sequences and PCR conditions for genotyping experiments.

\begin{tabular}{|c|c|c|c|c|}
\hline Genetic polymorphism & Primer sequence & Tm & Annealing temperature & PCR product size (bp) \\
\hline \multirow[t]{8}{*}{ FADS1 (rs174556) } & Forward inner primer (C allele): & $62.5^{\circ} \mathrm{C}$ & $57^{\circ} \mathrm{C}$ & 189 (C allele) \\
\hline & 5' - ACTGACTGTGATTACTATGACTGTGCTC-3' & & & \\
\hline & Reverse inner primer (T allele): & $62.5^{\circ} \mathrm{C}$ & & 268 (T allele) \\
\hline & 5'-ATGGGTGGAAGGAGAGTCTAGATTGA-3' & & & \\
\hline & Forward outer: & $62.5^{\circ} \mathrm{C}$ & & 404 (two outer primers) \\
\hline & 5' -TGAGCCTGTTATCTCATCTGTAAATGTGT-3' & & & \\
\hline & Reverse outer: & $62.5^{\circ} \mathrm{C}$ & & \\
\hline & 5'-GGGCCTTGTGAAGAAGTATATGAACTCT-3' & & & \\
\hline \multirow[t]{8}{*}{ FADS2 (rs99780) } & Forward inner primer ( $T$ allele): & $65^{\circ} \mathrm{C}$ & $59^{\circ} \mathrm{C}$ & 218 (T allele) \\
\hline & 5'-AATATTAACATGGGAAAAACCGCCCCGT-3' & & & \\
\hline & Reverse inner primer (C allele): & $66^{\circ} \mathrm{C}$ & & 297 (C allele) \\
\hline & 5'-AATATATGGAGCGTGGGAACCCGAGATG-3' & & & \\
\hline & Forward outer: & $65^{\circ} \mathrm{C}$ & & 460 (two outer primers) \\
\hline & 5'-TAATATAGGTITGTCTGGAGGCAGGGACTC-3' & & & \\
\hline & Reverse outer: & $64.5^{\circ} \mathrm{C}$ & & \\
\hline & 5'-ATATTATAGACGGACCTGTTGCCAAGCC-3' & & & \\
\hline \multirow[t]{8}{*}{ FADS3 (rs7115739) } & Forward inner (T allele): & $63^{\circ} \mathrm{C}$ & $61^{\circ} \mathrm{C}$ & 152 (T allele) \\
\hline & 5'-TATACCCGATCTTGGGCCTGATATTAGT-3' & & & \\
\hline & Reverse inner (G allele): & $63.5^{\circ} \mathrm{C}$ & & 260 (G allele) \\
\hline & 5'-TATTATTGACATTCTGGCTCCACCGC-3' & & & \\
\hline & Forward outer: & $64^{\circ} \mathrm{C}$ & & 358 (two outer primers) \\
\hline & 5'-TAATGTGGGTTGGGAGAACAGCTGAA-3' & & & \\
\hline & Reverse outer: & $63.5^{\circ} \mathrm{C}$ & & \\
\hline & 5'-ATGTACACTTCTCCTTCATGATCTCTCCC-3' & & & \\
\hline
\end{tabular}

allelic, dominant, recessive, codominant, overdominant, and logadditive models (Horita and Kaneko, 2015) through calculation of odds ratio (OR) and 95\% confidence intervals (CI). $P<0.05$ was considered as significant. For each SNP, a major allele (M) and a minor allele $(\mathrm{m})$ have been considered. Therefore, genotypes consisted include major allele homozygote (MM), heterozygote $(\mathrm{Mm})$, and minor allele homozygote $(\mathrm{mm})$. In the dominant model, comparison was made between MM and $\mathrm{Mm}+\mathrm{mm}$. In the recessive model, $\mathrm{MM}+\mathrm{Mm}$ were compared with $\mathrm{mm}$. In the overdominant model, it is assumed that the heterozygote has the most robust effect, thus it compares $\mathrm{MM}+\mathrm{mm}$ with $\mathrm{Mm}$. Codominant models such as additive and log-additive models assume that risk is increased/decreased in a stepwise manner from MM to $\mathrm{Mm}$ and to $\mathrm{mm}$ (Horita and Kaneko, 2015).

\section{RESULTS}

The current study included a total of 164 cases (75 women and 89 men) and 171 controls (81 women and 90 men). Table 3 shows the clinical variables assessed in cases and controls. Cases and controls were matched in all variables except for albumin levels, which were significantly lower among cases compared with controls, which may well be due to impaired renal function. However, such difference in albumin levels cannot affect genotypes. Thus, it was not necessary to match cases and controls in this parameter.
TABLE 3 | Clinical variables assessed in cases and controls.

\begin{tabular}{lcc}
\hline Variables & Cases & Controls \\
\hline TC (mean \pm SD, mg/dL) & $168.4 \pm 25.2$ & $172.4 \pm 21.2$ \\
HDL (mean l' SD, mg/dL & $42.56 \pm 1.79$ & $39.24 \pm 1.6$ \\
LDL (mean l' SD, mg/dL) & $108.27 \pm 3.65$ & $98.26 \pm 3.59$ \\
TG (mean l' SD, mg/dL) & $154.71 \pm 19.8$ & $159.67 \pm 19.2$ \\
Albumin (mean l' SD, g/dL) & $3.72 \pm 1.2$ & $5.21 \pm 1.6$ \\
BMl (mean l' SD, $\mathrm{kg} / \mathrm{m}^{2}$ ) & $23.1 \pm 1.5$ & $23.5 \pm 1.8$ \\
\hline
\end{tabular}

Genetic associations were assessed in different inheritance models (Horita and Kaneko, 2015). For rs174556, 47, 110, and 14 controls subjects had CC, CT, and TT genotypes, respectively. Among cases, these figures were 48, 107, and 9, respectively. For rs99780, 85, 69, and 17 control subjects were found to have CC, CT, and TT genotypes, respectively. A total of 88, 64, and 12 patients had CC, CT, and TT genotypes of rs99780, respectively. Finally, 134, 32, and 5 controls and 146, 17, and 1 patient(s) had GG, GT, and TT genotypes, respectively.

The rs174556 of FADS1 gene and rs99780 of FADS2 gene were not associated with risk of ESRD in any inheritance model. However, the rs7115739 of FADS3 was associated with risk of ESRD in all models except for recessive model. $T$ allele of this SNP was significantly less prevalent among cases compared with controls [OR $(95 \% \mathrm{CI})=0.44(0.25-0.77), P$ value $=0.004] . \mathrm{GT}$ and TT genotypes have been identified as protective genotypes against ESRD in the codominant model [OR $(95 \% \mathrm{CI})=0.49$ 
TABLE 4 | Results of association between FADS1-3 polymorphisms and ESRD.

\begin{tabular}{|c|c|c|c|c|c|c|c|}
\hline Gene & Locus & Model & Genotype & Controls & Patients & Odds ratio & $P$ value \\
\hline \multirow[t]{12}{*}{ FADS1 } & rs174556 & Allele & $\mathrm{C}$ & 204 (59.6\%) & 203 (61.9\%) & 1 & 0.55 \\
\hline & & & $\mathrm{T}$ & 138 (40.4\%) & 125 (38.1\%) & $0.91(0.67-1.24)$ & \\
\hline & & Codominant & $\mathrm{CC}$ & 47 (27.5\%) & 48 (29.3\%) & 1.00 & 0.61 \\
\hline & & & $\mathrm{CT}$ & $110(64.3 \%)$ & 107 (65.2\%) & $0.95(0.59-1.54)$ & \\
\hline & & & $\pi$ & $14(8.2 \%)$ & $9(5.5 \%)$ & $0.63(0.25-1.59)$ & \\
\hline & & Dominant & $\mathrm{CC}$ & 47 (27.5\%) & 48 (29.3\%) & 1.00 & 0.71 \\
\hline & & & $\mathrm{CT}+\mathrm{TT}$ & 124 (72.5\%) & 116 (70.7\%) & $0.92(0.57-1.47)$ & \\
\hline & & Recessive & $\mathrm{CC}+\mathrm{CT}$ & 157 (91.8\%) & 155 (94.5\%) & 1.00 & 0.33 \\
\hline & & & $\pi$ & $14(8.2 \%)$ & $9(5.5 \%)$ & $0.65(0.27-1.55)$ & \\
\hline & & Overdominant & $\mathrm{CC}+\pi$ & $61(35.7 \%)$ & 57 (34.8\%) & 1.00 & 0.86 \\
\hline & & & CT & $110(64.3 \%)$ & 107 (65.2\%) & $1.04(0.66-1.63)$ & \\
\hline & & Log-additive & & & & $086(0.59-1.27)$ & 0.46 \\
\hline \multirow[t]{12}{*}{ FADS2 } & rs99780 & Allele & C & 239 (69.9\%) & 240 (73.2\%) & 1 & 0.35 \\
\hline & & & $\mathrm{T}$ & $103(30.1 \%)$ & 88 (26.8\%) & $0.85(0.61-1.19)$ & \\
\hline & & Codominant & $\mathrm{CC}$ & $85(49.7 \%)$ & 88 (53.7\%) & 1.00 & 0.62 \\
\hline & & & CT & 69 (40.4\%) & 64 (39\%) & $0.90(0.59-1.54)$ & \\
\hline & & & $\pi$ & 17 (9.9\%) & $12(7.3 \%)$ & $0.68(0.31-1.51)$ & \\
\hline & & Dominant & $\mathrm{CC}$ & 85 (49.7\%) & 88 (53.7\%) & 1.00 & 0.47 \\
\hline & & & $\mathrm{CT}+\mathrm{TT}$ & 86 (50.3\%) & 76 (46.3\%) & $0.85(0.56-1.31)$ & \\
\hline & & Recessive & $\mathrm{CC}+\mathrm{CT}$ & 154 (90.1\%) & 152 (92.7\%) & 1.00 & 0.39 \\
\hline & & & $\pi$ & 17 (9.9\%) & $12(7.3 \%)$ & $0.72(0.33-1.55)$ & \\
\hline & & Overdominant & $\mathrm{CC}+\pi$ & 102 (59.6\%) & 100 (61\%) & 1.00 & 0.8 \\
\hline & & & $\mathrm{CT}$ & 69 (40.4\%) & 64 (39\%) & $0.95(0.61-1.47)$ & \\
\hline & & Log-additive & & & & $0.85(0.61-1.19)$ & 0.35 \\
\hline \multirow[t]{12}{*}{ FADS3 } & rs7115739 & Allele & $\mathrm{G}$ & 300 (87.7\%) & 309 (94.2\%) & 1 & 0.004 \\
\hline & & & $\mathrm{T}$ & 42 (12.3\%) & $19(5.8 \%)$ & $0.44(0.25-0.77)$ & \\
\hline & & Codominant & GG & 134 (78.4\%) & 146 (89\%) & 1.00 & 0.019 \\
\hline & & & GT & $32(18.7 \%)$ & 17 (10.4\%) & $0.49(0.26-0.92)$ & \\
\hline & & & $\pi$ & $5(2.9 \%)$ & $1(0.6 \%)$ & $0.18(0.02-1.6)$ & \\
\hline & & Dominant & $\mathrm{GG}$ & 134 (78.4\%) & 146 (89\%) & 1.00 & 0.0078 \\
\hline & & & $\mathrm{GT}+\mathrm{TT}$ & 37 (21.6\%) & $18(11 \%)$ & $0.45(0.24-0.82)$ & \\
\hline & & Recessive & $\mathrm{GG}+\mathrm{GT}$ & 166 (97.1\%) & 163 (99.4\%) & 1.00 & 0.095 \\
\hline & & & $\pi$ & 5 (2.9\%) & $1(0.6 \%)$ & $0.2(0.02-1.76)$ & \\
\hline & & Overdominant & $\mathrm{GG}+\pi$ & 139 (81.3\%) & 147 (89.6\%) & 1.00 & 0.029 \\
\hline & & & GT & 32 (18.7\%) & 17 (10.4\%) & $0.5(0.27-0.94)$ & \\
\hline & & Log-additive & & & & $0.47(0.27-0.82)$ & 0.005 \\
\hline
\end{tabular}

$(0.26-0.92)$ and OR $(95 \% \mathrm{CI})=0.18(0.02-1.6)$, respectively; $P$ value $=0.019]$. In the dominant model, GT + TT status was associated with lower risk of ESRD [OR (95\% CI) $=0.45(0.24-$ $0.82), P$ value $=0.0078]$. Assessment of association between this SNP and risk of ESRD in overdominant model revealed that GT genotype decreases risk of this condition [OR (95\% CI) $=0.5(0.27-0.94), P$ value $=0.029]$. Table 4 shows FADS1-3 polymorphisms and ESRD.

\section{DISCUSSION}

End-stage renal disease is a complicated condition resulting from several genetic and environmental factors (Obrador et al., 2017). Abnormalities in lipid metabolism including high levels of triglyceride, elevation in isodose and high-dense, triglyceride-rich particles, and decreases in high-density lipoprotein cholesterol have been reported in patients with chronic renal disorder (Gromovsky et al., 2018). Genes that regulate lipid metabolism might be involved in the pathogenesis of ESRD. In the current study, we investigated the association between FADS polymorphisms and ESRD in a population of Iranian patients. FADS genotypes have been previously found to be associated with inflammation and coronary artery disorder (Martinelli et al., 2008). Chronic inflammation has been regarded as a risk factor frequently detected in patients with ESRD patients and might lead to atherosclerotic changes (Stenvinkel and Alvestrand, 2002). Therefore, FADS genes might provide the mechanical link between inflammation, ESRD, and coronary artery disorder. Moreover, polymorphisms in the FADS gene cluster have been demonstrated to modulate fatty acid levels and affect the pathogenesis of atopic disorders (Lattka et al., 2009).

Based on the results of the current study, the rs174556 of FADS1 gene and rs99780 of FADS2 gene were not associated 
with the risk of ESRD in any inheritance model. However, the rs7115739 of FADS3 was associated with the risk of ESRD in all models except for the recessive model. $\mathrm{T}$ allele of this SNP was significantly less prevalent among cases compared with controls, therefore this allele can be regarded as a protective allele against ESRD. This SNP has been among variants identified in a scan of Inuit genomes for signatures of adaptation to an omega-3 polyunsaturated fatty acids-rich diet. Moreover, this SNP has been found to be associated with several metabolic and anthropometric features with a particularly large effect on weight and height (Fumagalli et al., 2015). Mechanistically, rs7115739 modulates fatty acid composition and might consequently influence the regulation of growth hormones (Fumagalli et al., 2015).

Consistently, GT and TT genotypes were found to decrease the risk of ESRD in the codominant model. In the dominant model, GT + TT status was associated with a lower risk of ESRD. Assessment of association between this SNP and risk of ESRD in the overdominant model revealed that the GT genotype decreases the risk of this condition. Therefore, the results of association analysis in different inheritance models consistently support the association between rs7115739 and the risk of ESRD. Although the underlying cause of the contribution of rs7115739 in the development of ESRD is not clear, the role of FADS genes in the modulation of inflammatory responses and the importance of these types of responses in the pathogenesis of ESRD suggest this mechanism as a possible way rs7115739 contributes in the pathogenesis of ESRD.

Taken together, the rs7115739 of FADS3 is suggested as putative modulator of the risk of ESRD in the Iranian population. The results of this study should be confirmed in larger cohorts of patients. Moreover, functional studies are required to clarify the mechanistical points. Our study has some limitations regarding the lack of validation of results from an independent external population, lack of evaluation of close SNP in LD with associated

\section{REFERENCES}

Campoy, C., Azaryah, H., Torres-Espínola, F. J., Martínez-Zaldívar, C., GarcíaSantos, J. A., Demmelmair, H., et al. (2021). Long-Chain Polyunsaturated Fatty Acids, Homocysteine at Birth and Fatty Acid Desaturase Gene Cluster Polymorphisms Are Associated with Children's Processing Speed up to Age 9 Years. Nutrients 13:131. doi: 10.3390/nu13010131

Chacon-Cortes, D., and Griffiths, L. R. (2014). Methods for extracting genomic DNA from whole blood samples: current perspectives. J. Bioreposit. Sci. Appl. Med. 2, 1-9. doi: 10.2147/bsam.s46573

Fumagalli, M., Moltke, I., Grarup, N., Racimo, F., Bjerregaard, P., Jørgensen, M. E., et al. (2015). Greenlandic Inuit show genetic signatures of diet and climate adaptation. Science 349, 1343-1347. doi: 10.1126/science.aab2319

Gorski, M., Van Der Most, P. J., Teumer, A., Chu, A. Y., Li, M., Mijatovic, V., et al. (2017). 1000 Genomes-based meta-analysis identifies 10 novel loci for kidney function. Sci. Rep. 7, 1-11.

Gromovsky, A. D., Schugar, R. C., Brown, A. L., Helsley, R. N., Burrows, A. C., Ferguson, D., et al. (2018). $\Delta-5$ Fatty Acid Desaturase FADS1 Impacts Metabolic Disease by Balancing Proinflammatory and Proresolving Lipid Mediators. Arterioscler. Thromb. Vasc. Biol. 38, 218-231. doi: 10.1161/atvbaha.117.30 9660 polymorphisms, and cross-validation with random subsets of the data. Moreover, this is a pilot study where we tested only three SNP. Thus, we plan to conduct a follow up study where we will investigate more polymorphisms in the same region.

\section{DATA AVAILABILITY STATEMENT}

The original contributions presented in the study are included in the article/supplementary material, further inquiries can be directed to the corresponding authors.

\section{ETHICS STATEMENT}

The studies involving human participants were reviewed and approved by the study protocol was approved by Ethical Committee of Shahid Beheshti University of Medical Sciences (IR. SBMU.UNRC.1398.10). The patients/participants provided their written informed consent to participate in this study.

\section{AUTHOR CONTRIBUTIONS}

MT and SG-F wrote the draft of the manuscript and revised the manuscript. AM performed the sample collection and clinical information. $\mathrm{KH}, \mathrm{MG}$, and $\mathrm{AA}$ performed the experiment. VK analyzed the data. All authors read and approved the submitted version.

\section{FUNDING}

The current study was supported by a grant from the Urology and Nephrology Research Center of Shahid Beheshti University of Medical Sciences.

Haghighi, A. N., Broumand, B., D’Amico, M., Locatelli, F., and Ritz, E. (2002). The epidemiology of end-stage renal disease in Iran in an international perspective. Nephrol. Dial. Transpl. 17, 28-32. doi: 10.1093/ndt/17.1.28

He, Z., Zhang, R., Jiang, F., Zhang, H., Zhao, A., Xu, B., et al. (2018). FADS1-FADS2 genetic polymorphisms are associated with fatty acid metabolism through changes in DNA methylation and gene expression. Clin. Epig. 10:113.

Horita, N., and Kaneko, T. (2015). Genetic model selection for a case-control study and a meta-analysis. Meta Gene 5, 1-8. doi: 10.1016/j.mgene.2015.04.003

Lattka, E., Illig, T., Heinrich, J., and Koletzko, B. (2009). FADS gene cluster polymorphisms: important modulators of fatty acid levels and their impact on atopic diseases. J. Nutrigenet. Nutrigenom. 2, 119-128. doi: 10.1159/000235559

Li, S. W., Wang, J., Yang, Y., Liu, Z. J., Cheng, L., Liu, H. Y., et al. (2016). Polymorphisms in FADS1 and FADS2 alter plasma fatty acids and desaturase levels in type 2 diabetic patients with coronary artery disease. J. Transl. Med. 14:79.

Lv, J. C., and Zhang, L. X. (2019). Prevalence and Disease Burden of Chronic Kidney Disease. Adv. Exp. Med. Biol. 1165, 3-15. doi: 10.1007/978-981-13-8871-2_1

Martinelli, N., Girelli, D., Malerba, G., Guarini, P., Illig, T., Trabetti, E., et al. (2008). FADS genotypes and desaturase activity estimated by the ratio of arachidonic acid to linoleic acid are associated with inflammation and coronary artery disease. Am. J. Clin. Nutr. 88, 941-949. doi: 10.1093/ajen/88.4.941 
Medrano, R. F., and de Oliveira, C. A. (2014). Guidelines for the tetra-primer ARMS-PCR technique development. Mole. Biotech. 56, 599-608.

Mousavi, S. S. B., Soleimani, A., and Mousavi, M. B. (2014). Epidemiology of endstage renal disease in Iran: a review article. Saudi J. Kidney Dis. Transpl. 25:697. doi: 10.4103/1319-2442.132242

Obrador, G. T., Schultheiss, U. T., Kretzler, M., Langham, R. G., Nangaku, M., Pecoits-Filho, R., et al. (2017). Genetic and environmental risk factors for chronic kidney disease. Kidney Int. Suppl. 7, 88-106.

Pattaro, C., Teumer, A., Gorski, M., Chu, A. Y., Li, M., Mijatovic, V., et al. (2016). Genetic associations at 53 loci highlight cell types and biological pathways relevant for kidney function. Nat. Comm. 7, 1-19.

Renal Du System (USRDS) (2010). Annual Data Report: Atlas of Chronic Kidney Disease and End-Stage Renal Disease in the United States. Bethesda, MD: National Institutes of Health, national Institute of Diabetes and Digestion Kidney Disease.

Satko, S. G., Freedman, B. I., and Moossavi, S. (2005). Genetic factors in end-stage renal disease. Kidney Internat. Suppl. 94, S46-S49.

Song, Z., Cao, H., Qin, L., and Jiang, Y. (2013). A case-control study between gene polymorphisms of polyunsaturated fatty acid metabolic rate-limiting enzymes and acute coronary syndrome in Chinese Han population. BioMed Res. Internat. 928178:2013.

Stenvinkel, P., and Alvestrand, A. (2002). Inflammation in end-stage renal disease: sources, consequences, and therapy. Semin. Dial. 15, 329-337. doi: 10.1046/j. 1525-139x.2002.00083.x

Vaziri, N. D. (2009). Causes of dysregulation of lipid metabolism in chronic renal failure. Semin. Dial. 22, 644-651. doi: 10.1111/j.1525-139x.2009.00661.x

Wu, Y., Zeng, L., Chen, X., Xu, Y., Ye, L., Qin, L., et al. (2017). Association of the FADS gene cluster with coronary artery disease and plasma lipid concentrations in the northern Chinese Han population. Prostaglandins leukot. Essent. Fatty Acids 117, 11-16. doi: 10.1016/j.plefa.2017.01.014

Wuttke, M., and Köttgen, A. (2016). Insights into kidney diseases from genomewide association studies. Nat. Rev. Nephrol. 12, 549-562. doi: 10.1038/nrneph. 2016.107

Xie, L., and Innis, S. M. (2008). Genetic variants of the FADS1 FADS2 gene cluster are associated with altered (n-6) and (n-3) essential fatty acids in plasma and erythrocyte phospholipids in women during pregnancy and in breast milk during lactation. J. Nutr. 138, 2222-2228. doi: 10.3945/jn.108.09 6156

Conflict of Interest: The authors declare that the research was conducted in the absence of any commercial or financial relationships that could be construed as a potential conflict of interest.

Publisher's Note: All claims expressed in this article are solely those of the authors and do not necessarily represent those of their affiliated organizations, or those of the publisher, the editors and the reviewers. Any product that may be evaluated in this article, or claim that may be made by its manufacturer, is not guaranteed or endorsed by the publisher.

Copyright (C) 2021 Miladipour, Gholipour, Honarmand Tamizkar, Abak, Kholghi Oskooei, Taheri and Ghafouri-Fard. This is an open-access article distributed under the terms of the Creative Commons Attribution License (CC BY). The use, distribution or reproduction in other forums is permitted, provided the original author(s) and the copyright owner(s) are credited and that the original publication in this journal is cited, in accordance with accepted academic practice. No use, distribution or reproduction is permitted which does not comply with these terms. 